\title{
2D FSI determination of mechanical stresses on aneurismal walls
}

\author{
Natalia Veshkina ${ }^{\mathrm{a}}$, Ireneusz Zbicinski ${ }^{\mathrm{a},{ }^{*}}$ and Ludomir Stefańczyk ${ }^{\mathrm{b}}$ \\ ${ }^{a}$ Faculty of Process and Environmental Engineering, Lodz Technical University, 213 Wólczańska \\ St.,90-924 Lodz, Poland \\ ${ }^{b}$ Department of Radiology and Diagnostic Imaging Medical University of Lódź, 22 Kopcińskiego Str., \\ 90-153 Lodz, Poland
}

\begin{abstract}
In this study, a fluid-structure interaction analysis based on the application of patient-specific mechanical parameters of the aneurismal walls was carried out to predict the rupture side during an abdominal aortic aneurysm (AAA). Realistic geometry of the aneurysm was reconstructed from CT data acquired from the patient, and patient-specific flow conditions were applied as boundary conditions. A newly developed non-invasive methodology for determining the mechanical parameters of the patient-specific aortic wall was employed to simulate realistic aortic wall behaviors. Analysis of the results included time-averaged wall shear stress (TAWSS), oscillatory shear index (OSI), and von Mises stress (VMS). Results of the TAWSS, OSI, and VMS were compared to identify the most probable region of the AAA's rupture. High OSI, which identified the region of wall degradation, coincided with the location of maximum VMS, meaning that the anterior part of the aneurismal wall was a potential region of rupture.
\end{abstract}

Keywords: Fluid-structure interaction, abdominal aortic aneurysm, mechanical parameters of the aortic wall, computed tomography, computational fluid dynamics

\section{Introduction}

An abdominal aortic aneurysm is a life threatening disease, causing $1.3 \%$ of all deaths among men above 65 in developed countries [1,2]. Generally, abdominal aortic aneurysms are related to the degradation of the elastic tunica media, but may also be caused by genetic disorders such as Marfan syndrome and Ehlers-Danlos syndrome or due to smoking, hypertension, and atherosclerosis [3]. The initial stage in the formation of an aneurysm involves structural changes as a result of a degenerative process in the vascular wall. After these changes occur, the blood flow patterns are modified, leading to alterations in the wall's shear stress. The dynamic relationship between the geometric and hemodynamic factors eventually leads to further thinning of the aortic wall and an aneurismal rupture [4].

\footnotetext{
*Corresponding author: Ireniusz Zbicinski, Faculty of Process and Environmental Engineering, Lodz Technical University, 213 Wólczańska St., 90-924 Lodz, Poland. Tel.: +48 42 6313773; Fax: $+48 \quad 42$ 636-56-63; E-mail: ireneusz.zbicinski@p.lodz.pl.
}

0959-2989/14/\$27.50 @ 2014 - IOS Press and the authors. 
The current method of calculating the risk of rupture is based on assessing the size of the aneurysm or its maximum diameter. If the diameter of an AAA exceeds $5.5 \mathrm{~cm}$, the risk of rupture increases significantly. However, this method based on size assessment is not reliable, since some AAAs rupture at sizes smaller than $5 \mathrm{~cm}$, and others can grow to as large as $9 \mathrm{~cm}$ without rupturing. Some researchers suggest that the failure of abdominal aortic aneurysm walls is not related to the AAA diameter [5]. Recent studies on AAAs have shown that the peak wall stress is a more reliable indicator of the risk of rupture. Miller [6] reported that AAAs can develop in conditions of oxidative stress, where reactive oxygen species (ROS) mediate the activation of matrix degradation proteins, which leads to cell apoptosis and results in the loss of elasticity and strength in arterial walls. High wall shear stress caused by movement of blood reduces ROS by increasing the expression of an oxygenase that has anti-inflammatory effects, helping to reduce the oxidative reaction [7]. However, to fully understand aneurismal ruptures, it is not enough to consider only wall mechanics (wall stresses) or hemodynamic factors (oscillatory shear index (OSI)). It is also necessary to estimate the dynamic interaction between the blood flow and wall displacement in AAAs [4]. The recent approach, which allows estimating both the hemodynamic forces of the blood flow and the stresses occurring inside the aortic wall, is based on fluid-structure interaction (FSI) analysis. A number of studies have been carried out on the implementation of FSI analysis to estimate the risks of aneurismal ruptures; however, the studies were not based on patient-specific mechanical parameters of aortic walls, but rather represented a general approach. Reliable FSI models for the purposes of clinical diagnostics require patient-specific values of the material parameters. In this work, FSI analysis based on calculated non-invasive patient-specific values of the material parameters of the aortic walls were carried out.

To determine patient-specific material parameters, an experimental set-up, which reflects blood flow in human arteries, was built and tested. The set-up allowed for measuring the changes in the diameter of a silicone pipe, which represented the aorta, and the input and output parameters for constant and pulsating flow. Based on the acquired experimental data, the FSI computational model was elaborated on to describe the mutual response of the solid and fluid phases simultaneously. An inverse analysis method was used to fit the numerically modeled oscillations of the pipe diameter to the experimental values obtained in relevant conditions and determine the mechanical parameters for the silicone.

A full description of the methodology for the non-invasive determination of the patient-specific material parameters of the aortic walls is presented in [8].

\section{Methods}

\subsection{Patient - specific model reconstruction}

The model of the AAA was reconstructed from computed tomography (GE Medical Systems, number of slices 759 , slice thickness $0.625 \mathrm{~mm}$ ) images of an 88-year old patient with a developed aneurysm in the descending abdominal aorta. The maximum aneurismal diameter, measured at a maximum cross-sectional dimension, was $9.5 \mathrm{~cm}$. The segmentation of the images and 3D geometry reconstruction were accomplished using Materialise's Interactive Medical Image Control System (MIMICS 14.0), which made it possible to process the 2D image data (CT data) to construct a 3D model with the utmost accuracy. 


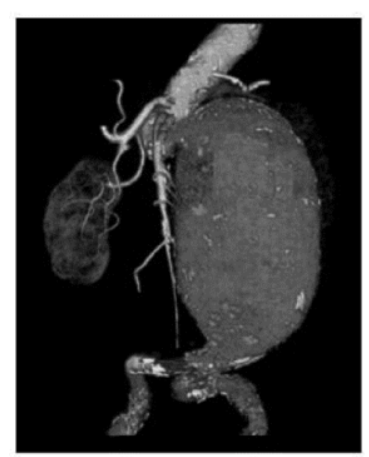

(a)

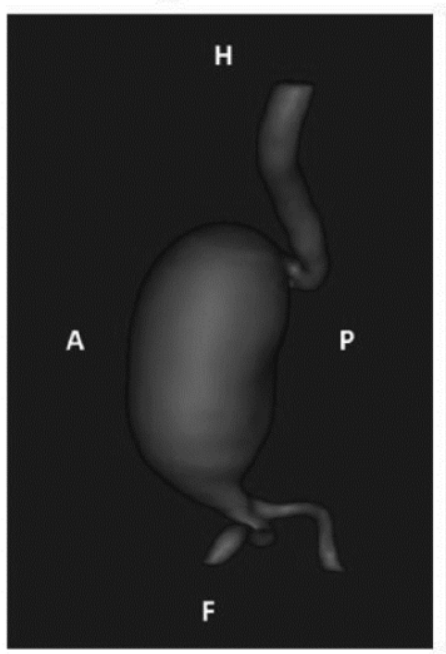

(c)

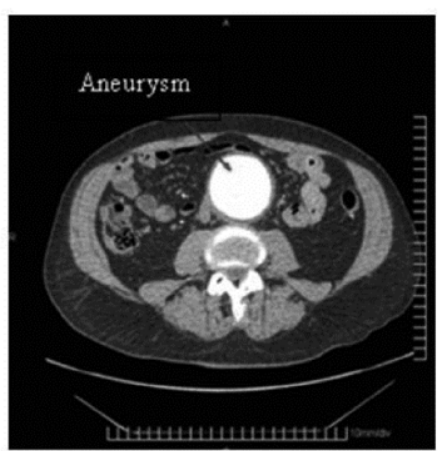

(b)

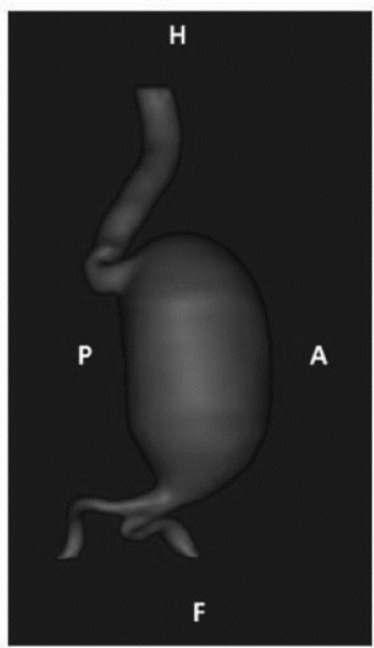

(d)

Fig. 1. The patient-specific abdominal aortic aneurysm model obtained from a CT image: (a) an oblique sagittal view; (b) a transaxial view; (c) and (d) the reconstructed geometry. Data provided by the Department of Radiology and Diagnostic Imaging, Medical University of Lodz, Poland.

The images were stored in DICOM format (Digital Imaging and Communication in Medicine) and then were imported into MIMICS 14.0 to reconstruct the fluid part of the 3D model of the aneurysm. A smoothing procedure was applied to avoid artifacts from the scanning data. Changes in the volume of the smoothed geometry did not exceed 5\% of the initial volume of geometry, which ensured that the smoothing procedure did not affect the accuracy of the AAA geometry reconstruction.

The results of the segmentation are shown in Figure 1, where (a) shows an oblique sagittal view of the aneurysm, (b) shows a transaxial view of the aneurysm, and (c) and (d) represent the reconstructed AAA geometry. The wall thickness of AAA was assumed to be equal to $1 \mathrm{~mm}$ according to literature experimental data. No intra luminal thrombus (ILT) was present inside the aneurysm.

\subsection{Boundary conditions for fluid part}

The flow information at the inlet of the AAA was obtained using the speckle tracking USG technique in the descending part of the aorta. Based on these data, the blood flow velocity waveform was reproduced and used as inlet boundary conditions (see Figure 2). 


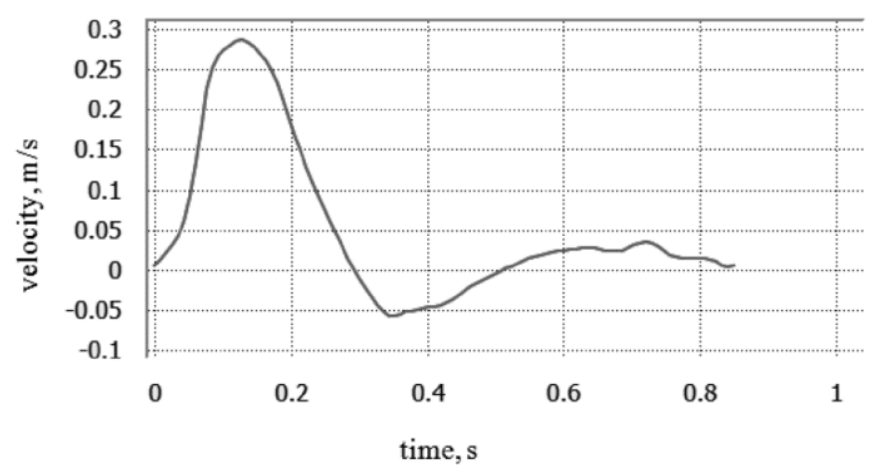

Fig. 2. Average blood flow velocity waveform.

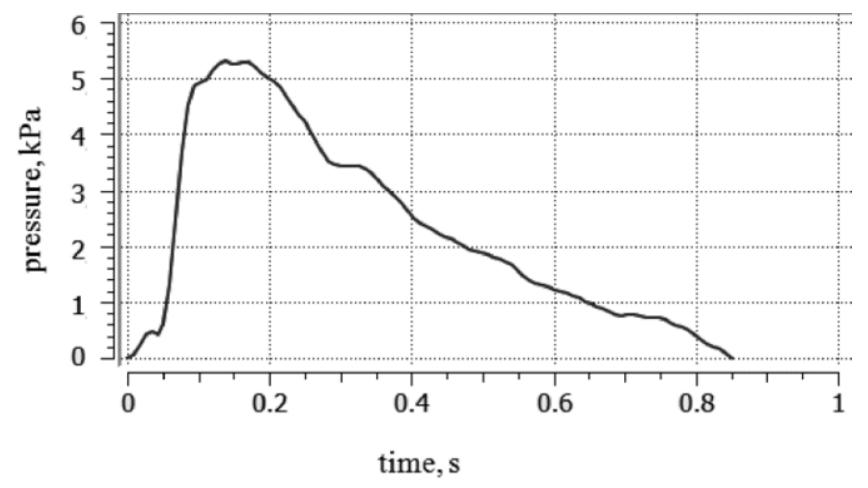

Fig.3. Maximum pressure waveform applied in the simulations [9].

Blood flow was assumed to be turbulent and an inlet turbulence level of $1.5 \%$ was specified. At the outlets of the right and left iliac arteries, pulse pressure waveforms were imposed. Patient-specific pressure information, a pressure waveform, was taken from literature [9]. Figure 3 represents the maximum pressure waveform applied for the simulations.

A phase shift of $0.03 \mathrm{~s}$ between the velocity and pressure peaks was set in the calculations, which corresponded to the real physiological value. No-slip boundary conditions were applied on the aortic wall. Blood was considered an incompressible, homogeneous, Newtonian fluid with a density equal to $1060 \mathrm{~kg} / \mathrm{m}^{3}$ and dynamic viscosity of $0.0034 \mathrm{~Pa}^{*} \mathrm{~s}$.

\subsection{Boundary conditions for structural part}

In order to run the FSI simulation, the input file which contains the information on mechanical properties of the aortic wall, type of constraints and simulation settings, was specified. To simulate the realistic pulsatile aortic wall movement, the following boundary conditions and constraints were applied:

- The ends of the AAA were constrained in the Z-direction (axial direction), although movements in the X- and Y-directions (radial and circumferential directions) were allowed to simulate more realistic movements of the aorta. To avoid convergence problems, some extra nodes at the ends of the geometry were additionally fixed in the X-and Y-directions. The loads applied on the internal 
surface of the aortic wall were transferred from CFD part of the model and represented the pressure difference between the systolic and diastolic phase.

- An AAA wall was modeled as an incompressible, isotropic, homogenous, hyperelastic material using a finite strain constitutive model proposed by [10]. A Mooney-Rivlin solid model was used for the arterial wall with a strain energy density function described by Eq. (1):

$$
\mathrm{W}=\alpha\left(\mathrm{I}_{\mathrm{B}}-3\right)+\beta\left(\mathrm{I}_{\mathrm{B}}-3\right)^{2}
$$

where $\mathrm{W}$ is the strain energy density function, IB is the first invariant of the left Cauchy-Green tensor B. The model parameters $\alpha$ and $\beta$ are the material parameters, which were calculated using a noninvasive approach described in $\mathrm{PhD}$ thesis (Veskhina, 2013). The model parameters were set to $\alpha=$ $12.00 \mathrm{~N} / \mathrm{cm} 2$ and $\beta=90.00 \mathrm{~N} / \mathrm{cm} 2$.

\subsection{Fluid-Structure Interaction settings}

To perform the completed analysis of the blood flow hemodynamics and stress analysis of the AAA, a coupled FSI model was expanded. The fluid and solid domains were coupled using ANSYS 14.1 commercial software. The 2-way FSI analysis was performed using the finite volume method implemented into ANSYS commercial software. The structural and fluid equations were solved independently and then loads were transferred between two solvers after each converged time step. The exchanged information included the pressure from the fluid part and the wall displacement from the structural part. Pressure was calculated in Ansys CFX in the field loop until a defined convergence level was achieved or the maximum number of coefficient loops was reached. Then, this value was transferred to the ANSYS structural on the interface defined previously. Three cardiac cycles were calculated to ensure periodicity; a coupled time step was equal to $0.01 \mathrm{~s}$. In the analysis of the fluid flow, a maximum RMS residual of $10^{-6}$ was set to control solution stability, and approximately 40 interactions were needed to reach convergence at each time step. In the structural analysis, the maximum RSM residual was defined as $10^{-3}$ with 15 iterations.

\section{Results and discussion}

Analysis of the results included a comparison of the wall shear stress (WSS), oscillatory shear index (OSI), and von Mises Stress (VMS).

\subsection{Wall shear stress analysis}

During unsteady and pulsatile blood flow, the velocity vectors and related WSS vectors changed magnitude and direction, therefore time-averaged WSS (TAWSS) was used to estimate the mean shear stress acting on the lumen surface during the cardiac cycle:

$$
T A W S S=\frac{1}{T} \int_{0}^{T}\left|\tau_{\omega}\right| d t
$$



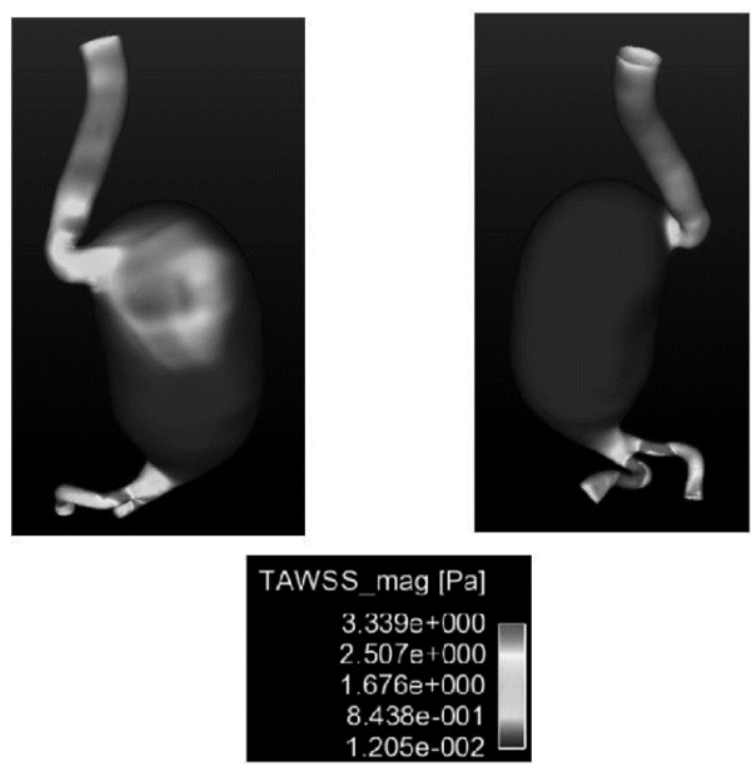

Fig. 4. Time-averaged wall shear stress (TAWSS) contours of the AAA.

where $\mathrm{T}$ is the cardiac cycle period and $\tau_{\omega}$ is the instantaneous wall shear stress. Figure 4 shows the time-averaged wall shear stress (TAWSS) contours of the AAA.

Regions of high TAWSS were observed in the coarctation region as well as in the iliac arteries, where the velocity was high. A jet of fluid from coarctation impinged on the surface of the aneurysm, inducing flow recirculation, which affected the shear stress values along the wall. A large region of low TAWSS was found along the anterior surface of the aneurysm, corresponding to the low velocity inside the aneurismal sack.

In this case, disturbances of the flow were explained by the presence of the combined temporal and spatial retardations of the flow, as opposed to just temporal retardations during the deceleration phase of the heart cycle, as seen in normal blood vessels. The sudden increase in the cross-sectional area inside the aneurysm caused flow separation, flow reversal, and adverse pressure gradients, effects similar to a jet in which the recirculated flow between the jet and the wall is sluggish, resulting in a reduced wall shear stress. However, in the flow reattachment region, the WSS can be amplified.

\subsection{Oscillatory shear index}

The OSI, which gives the information on the cyclic variation of WSS directions, was also analyzed. Figure 5 shows that high OSI was present along the anterior side of the aneurismal wall. Flow recirculation in this area resulted in low TAWSS and high temporal oscillation of wall shear stress, while the location of low OSI distribution corresponded to the areas with high TAWSS values.

The areas with high OSI values are the most risky as far as an aneurysm rupture is concerned. High OSI values cause wall degradation due to cell apoptosis and can lead to the aneurismal ruptures. 

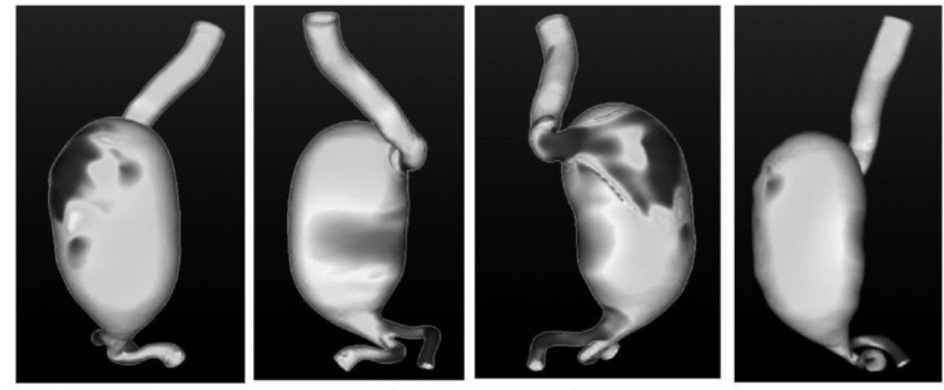

OSI

$5.000 \mathrm{e}-001$
$3.750 \mathrm{e}-001$

$3.750 \mathrm{e}-001$
$2.500 \mathrm{e}-001$

(250e-001

Fig. 5. Distribution of oscillatory shear index (OSI) along the aneurismal wall.

\subsection{Von Mises stress analysis}

The results of the structural analysis are presented in terms of von Mises stress (VMS) formulation, which expresses the global distortion experienced by the aortic wall. VMS is often used as a criterion for failure of a material.

Figure 6 shows the von Mises stress distribution on the outer wall of the aneurysm at peak pressure. Higher von Mises stress was located in the bulbous part of the aneurysm, with the maximum value of VM stress equal to $63 \mathrm{kPa}$. By comparing the location of maximum OSI that acts from the blood side and maximum von Mises stress that experiences the aneurismal wall, a clear interaction was observed. High OSI, which points out the region of wall degradation, coincides with the location of maximum VMS, meaning that the anterior part of the aneurismal wall is a potential region of rupture.

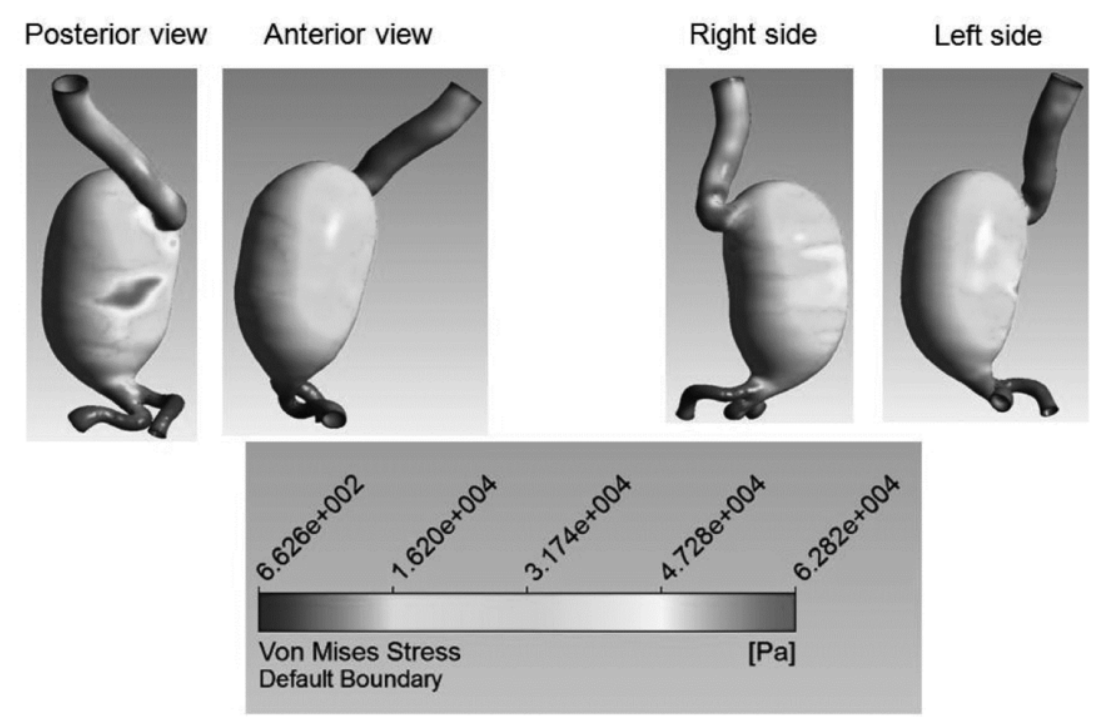

Fig. 6. Von Mises stress distribution on the outer wall of the AAA at peak pressure. 


\section{Conclusion}

The stress and flow patterns of a patient with an AAA were analyzed using a fluid-structure interaction method based on the application of patient-specific mechanical parameters of the aneurismal wall.

High OSI and von Mises stress values were found in the aneurismal bulge, indicating the area with an elevated probability of rupture due to cell apoptosis. The results of TAWSS, OSI, and VMS were compared to identify the most probable region of the AAA's rupture. An interaction between the hemodynamic blood flow factors and stresses occurring inside the aortic wall was observed, showing evidence that extensive fluid-structure interaction analysis based on patient-specific data, such as mechanical parameters of the aortic wall and real geometry of the aorta, provided a reliable assessment of the rupture risk of the abdominal aortic aneurysm.

\section{Acknowledgement}

This work was supported by the National Science Center of Poland (grant number 2011/01/B/ST8/06689). The authors thank Professor X.Y. Xu and Professor N.B. Wood from Imperial College London for helpful discussion and brilliant advices.

\section{References}

[1] N. Sakalihasan, R. Limet and O.D. Defawe, Abdominal Aortic Aneurysm, Seminar Lancet 365 (2005), 1577-1589.

[2] D. Epstein, M.J. Sculpher, J.T. Powell, S.G. Thompson, L.C. Brown and R.M. Greenhalgh, Long-term costeffectiveness analysis of endovascular versus open repair for abdominal aortic aneurysm based on four randomized clinical trials, British Journal of Surgery 101 (2014), 623-631.

[3] F.P.P. Tan, R. Torii, A. Borghi, R.H. Mohiaddin, N.B. Wood and X.Y. Xu, Fluid-structure interaction analysis of wall stress and flow patterns in a thoracic aortic aneurysm. International Journal of Applied Mechanics 1 (2009), 179-199.

[4] S.S. Raut, A. Jana, V. De Oliveira, S.C. Muluk and E.A. Finol, The importance of patient-specific regionally varying wall thickness in abdominal aortic aneurysm biomechanics, J. Biomech. Eng. 135 (2013), 081010.

[5] D.A. Vorp, B.J. Schiro, M.P. Ehrlich, T.S. Juvonen, M.A. Ergin and B.P. Griffith, Effect of ameurysm on the tensile strength and biomechanical behavior of the ascendingthoracic aorta, The Annals of Thoracic Surgery 75 (2003), 12101214.

[6] F.J. Jr. Miller, Aortic aneurysms: It's all about stress, Arteriosclero. Thromb. Vasc. Biol. 22 (2002), 1948-1949.

[7] T.K. Nakahashi, K. Hoshina, P.S. Tsao, E. Sho, J.K. Karwowski, C. Yeh, R.B. Yang, J.N. Topper and R.L. Dalman, Flow loading induced macrophage antioxidative gene expression in experimental aneurysms, Arterioscler. Thromb. Vasc. Biol. 22 (2002), 2017-2022.

[8] N. Veshkina, Determination of hemodynamics in collapsible walls, Ph.D. Dissertation, Lodz University of Technology, 2013.

[9] M.S. Olufsen and C.S. Peskin, Numerical simulation and experimental validation of blood flow in arteries with structured-tree outflow conditions, Biomed. Eng. 28 (2000), 1281-1299.

[10] M.L. Raghavan and D.A. Vorp, Toward a biomechanical tool to evaluate rupture potential of abdominal aortic aneurysm: Identification of a finite strain constitutive model and evaluation of its applicability, J. Biomech. 33 (2000), 475-482. 